\title{
Nail Gun Injury to the Left Ventricle Causing Coronary Pulmonary Fistula, A Case Report
}

Turki Albacker ${ }^{1}$, Omar BinSebayel ${ }^{1}$, Ali Alzahrani ${ }^{1}$, Ammar Almansour ${ }^{1}$, Aljoud Algazlan ${ }^{1}$, Faisal Alqumaizi ${ }^{1}$, Abdulelah Alqahtani ${ }^{1}$, Ahmed Eldemerdash ${ }^{1}$, and Bakir Bakir ${ }^{1}$

${ }^{1}$ College of Medicine, King Fahad Cardiac Center, King Saud University Medical City, King Saud University

April 30, 2021

\begin{abstract}
We present a case of a young patient who presented with unstable angina due to coronary-pulmonary fistula caused by a nail-gun injury that stayed asymptomatic for 3 years. The patient has a successful surgical resection of the fistula and removal of the foreign body.
\end{abstract}

Chronic Nail Gun Injury to the Left Ventricle Causing Coronary Pulmonary Fistula, A Case Report

Running Head: Coronary Fistula due to Nail Gun Cardiac Injury

Authors:

Turki B. Albacker, Omar Alsubayel, Ali Alzahrani, Ammar Almansour, Aljoud Alqazlan, Faisal Algumaizy, Abdulilah Alqahtani, Ahmed El-Demerdash, Bakir Bakir

Institutions:

Cardiac Sciences Department, College of Medicine, King Fahad Cardiac Center, King Saud University Medical City, King Saud University, Riyadh, Saudi Arabia

Key words: Nail Gun, Injury, Coronary, Pulmonary, Fistula

Word Count: 1448 words

Abstract word count: 44 words

Corresponding Author:

Turki B. Albacker, MBBS, M.Sc, FRCSC, FACS, FACC

Associate Professor of Cardiac Sciences

Cardiac Surgery Division, Cardiac Sciences Department, College of Medicine

Consultant Cardiac \& Aortic Surgeon, King Fahad Cardiac Center

King Saud University Medical City, King Saud University

Riyadh, Saudi Arabia

Tel: +966-1-4679353 
Fax: $+966-1-4671581$

\section{Chronic Nail Gun Injury to the Left Ventricle Causing Coronary Pulmonary Fistula, A Case Report}

\section{Summary}

We present a case of a young patient who presented with unstable angina due to coronary-pulmonary fistula caused by a nail-gun injury that stayed asymptomatic for 3 years. The patient has a successful surgical resection of the fistula and removal of the foreign body.

\section{Chronic Nail Gun Injury to the Left Ventricle Causing Coronary Pulmonary Fistula, A Case Report}

\section{Introduction}

Nail guns had been widely used in the industrial field due to the effectiveness and time saving properties [1], but the lack of proper training resulted in numerous body injuries mainly in peripheries [2]. However, heart related nail gun injuries are rare and mostly to the right chambers of the heart [3]. The majority of cases ended up with good outcomes despite their acute presentations to the ED. This favorable prognosis is mainly attributed to the presence of experts who will guide the management approach starting from the preoperative imaging and surgical intervention. We present a rare case of a chronic nail gun injury to the left ventricle with formation of coronary-pulmonary artery fistula.

\section{Case presentation}

Institutional review board approval for reporting this case and the need for informed consent were waived as per the institution regulation An otherwise healthy, 32-year-old male was admitted electively to our department with chest pain and shortness of breath on moderate exertion that was progressing with time over one year. His initial chest X-Ray showed a foreign body at the apex of the heart. On inquiry, the patient gave a history of Nail Gun Injury to the chest four years ago, which caused a very small wound in the left side of the chest that had minimal bleeding and some pain for two days that was relieved by pain killers. He did not seek any medical advice afterward since the pain resolved and he continued to be asymptomatic until one year ago when he started to have chest pain and shortness of breath on severe exertion that progressed over the year to occur with moderate exertion. Echocardiogram showed preserved left ventricular function with a hyperechoic foreign body at the posterior basal part of the left ventricle (Figure 1). All valves had normal structure and function. Computerized Tomography (CT) of the chest with contrast was performed to better understand the exact position of the foreign body and its relation to the surrounding structures. The chest CT showed a long narrow white shadow (measuring $5 \mathrm{~cm}$ ) penetration the medial part of the left lung and the posterior basal part of the left ventricle with a fibrous band surrounding the foreign body (Figure 2 ). Coronary angiography was performed to rule out any coronary injury given the patient presentation with unstable angina. The coronary angiogram revealed a fistula between the tip of posterolateral branch of the right coronary artery and the left lower lobe posterior basal segmental branch of the left pulmonary artery (Figure 3).

The decision was made to take the patient to the OR for removal of the foreign body and ligation of the coronary pulmonary fistula. After median sternotomy, the pericardium was then opened, which interestingly showed no bleeding or effusion. The heart was left for examination and showed a thick fibrous band that 2 $\mathrm{cm}$ wide connecting the posterior basal part of the left ventricle and the posterior pericardium, Heparin was administered and cardiopulmonary bypass (CPB) was established using ascending aortic cannulation and two-stage venous cannulation. The heart was drained and the operation was performed on a beating heart. The fibrous band was resected circumferentially off the posterior pericardium and revealed the nail that was protruding from the medial part of the left lung. The nail (Figure 4) was pulled off the lung tissues and then the other end was pulled off the left ventricular wall with difficulty. The two ends of the fibrous band on both

sides (the heart and the posterior pericardium and left pleura) that are containing the bridging collaterals of 
the fistula were ligated using 2 U-shaped 3-0 prolene mattress sutures supported with pledgets (Figure 5). The patient had non-eventful postoperative course and was discharged home after 4 days in stable condition.

\section{Discussion}

The majority of coronary artery fistulae are asymptomatic as they are hemodynamically not significant and are incidentally identified by coronary angiography, CT angiogram, echocardiogram or multi-detector row computed tomography (MDCT) with 3D reconstruction.[4]. Penetrating chest injuries causing coronary artery fistula are rare [5]. Moreover, presentation of patients may differ from lack of symptoms to heart failure or pulmonary hypertension [6,7]. However, most fistulas are hemodynamically non-significant with CT angiogram, echocardiogram or multi-detector row computed tomography (MDCT) as a diagnostic method [4]. We presented the first case of nail gun injury to the left ventricle with the coronary artery fistula to the left pulmonary artery presenting with unstable angina presenting after 4 years of asymptomatic course.

PubMed database was reviewed for relevant English literature from 1980 to 2020 using the keywords "nail gun", "coronary artery fistula", and "cardiac trauma". Twenty-three cases of nail gun injury to the heart were found, with right chambers most commonly involved as penetration site (Table 1.). Unlike our patient, most cases presented acutely and required urgent surgical removal of the nail. Chest x-ray, CT, intraoperative transesophageal echocardiogram were used as modality of choice for diagnosis of patients and guidance throughout the management plan. However, due to the presentation of our patients with unstable angina and his chronic presentation, we decided to do a coronary angiogram which revealed abnormal vascular mesh between the right coronary artery and the left pulmonary artery.

Treatment approach depends on the case presentation; either the thoracotomy or median sternotomy are acceptable. However, the choice is mainly guided by the hemodynamic status and the location of the penetration. There are few cases in which both median sternotomy and CPB that has the advantage of providing a controlled field that allows for manipulation and movement of the heart without the risk of circulatory compromisation or arrhythmia. In this case We used CPB with median sternotomy without cardioplegia.

Traumatic coronary artery fistulae due to penetrating chest injuries are rare . Patients may present with congestive heart failure, pulmonary hypertension, or endocarditis. However, most patients may stay asymptomatic $[6,7]$. Treatment options for symptomatic patients may include (1) surgical correction with ligation of feeding vessels of the the coronary artery fistula [8] with or without bypass grafting of the distal vessel, (2) percutaneous closure either with coil embolization, or covered stent [9]. However, there are currently no well-designed guidelines for deciding whether and how to treat a coronary-pulmonary artery fistula.

\section{Conclusion}

Cardiac Nail Gun injury can stay asymptomatic for long period of time and can present later with coronary fistula that can be treated successfully with open surgical resection of the fistula and removal of the foreign body.

\section{References}

1. Nölke L, Naughton P, Shaw C, Hurley J, Wood AE. Accidental nail gun injuries to the heart: diagnostic, treatment, and epidemiological considerations. J Trauma Acute Care Surg . 2005;58(1):172-174.

2. Prevention C for DC and. Nail-gun injuries treated in emergency departments-United States, 2001-2005. MMWR Morb Mortal Wkly Rep . 2007;56(14):329-332.

3. Jodati A, Safaei N, Toufan M, Kazemi B. A unique nail gun injury to the heart with a delayed presentation. Interact Cardiovasc Thorac Surg . 2011;13(3):363-365.

4. Bagai A, Lu J, Al-Amro B, et al. Management of right coronary artery fistula caused by nail gun trauma. J Thorac Cardiovasc Surg . 2016;152(6):e139-e141. 
5. Eren E, Keles C, Sareyyupoglu B, Bozbuga N, Balkanay M, Yakut C. Penetrating injury of the heart by a nail gun. $J$ Thorac Cardiovasc Surg . 2004;127(2):598.

6. Thandroyen FT, Matisonn RE. Penetrating thoracic trauma producing cardiac shunts. J Thorac Cardiovasc Surg . 1981;81(4):569-573.

7. Liberthson RR, Sagar K, Berkoben JP, Weintraub RM, Levine FH. Congenital coronary arteriovenous fistula. Report of 13 patients, review of the literature and delineation of management. Circulation . 1979;59(5):849-854.

8. Narh JT, Zahid E, Shivaraj K, Sahni S, Kariyanna PT, Khan A. Steal and strain: A case of coronary artery fistula presenting with coronary steal syndrome and underlying bronchiectasis. Respir Med Case Reports . 2020;31:101301.

9. Pritts TA, Knight D, Davis BR, Porembka D, Cuschieri J. Accidental self-inflicted nail gun injury to the heart. Inj extra . 2005;36(11):517-519.

Table 1. Cases of nail gun penetration the heart.

Figure 1: Echocardiogram showing a hyperechoic foreign body at the posterior basal part of the left ventricle.

Figure 2: Chest CT showing a long narrow white shadow (measuring $6 \mathrm{~cm}$ ) penetration the medial part of the left lung and the posterior basal part of the left ventricle. A) 3D reconstructed image, B) Axial Image

Figure 3: A) Coronary angiogram showing a fistula between the tip of posterolateral branch of the right coronary artery and the left lower lobe posterior basal segmental branch of the left pulmonary artery, B) A magnified image showing the relationship of the nail to the fistula, C) Showing the inflow and outflow arteries for the fistula.

Figure 4: The Gun-nail measuring $5 \mathrm{~cm}$.

Figure 5: The fibrous band that are containing the bridging collaterals of the fistula was ligated using 2 U-shaped 3-0 prolene mattress sutures supported with pledgets

Figure 1:

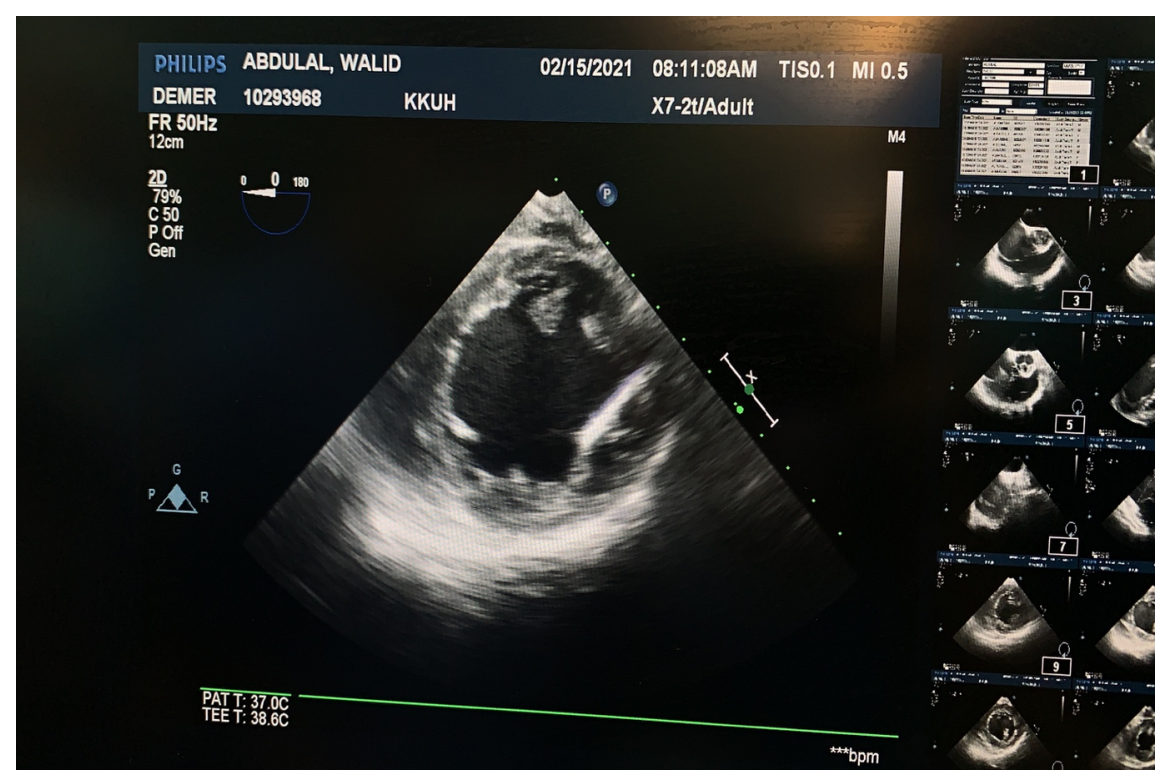

Figure 2: 


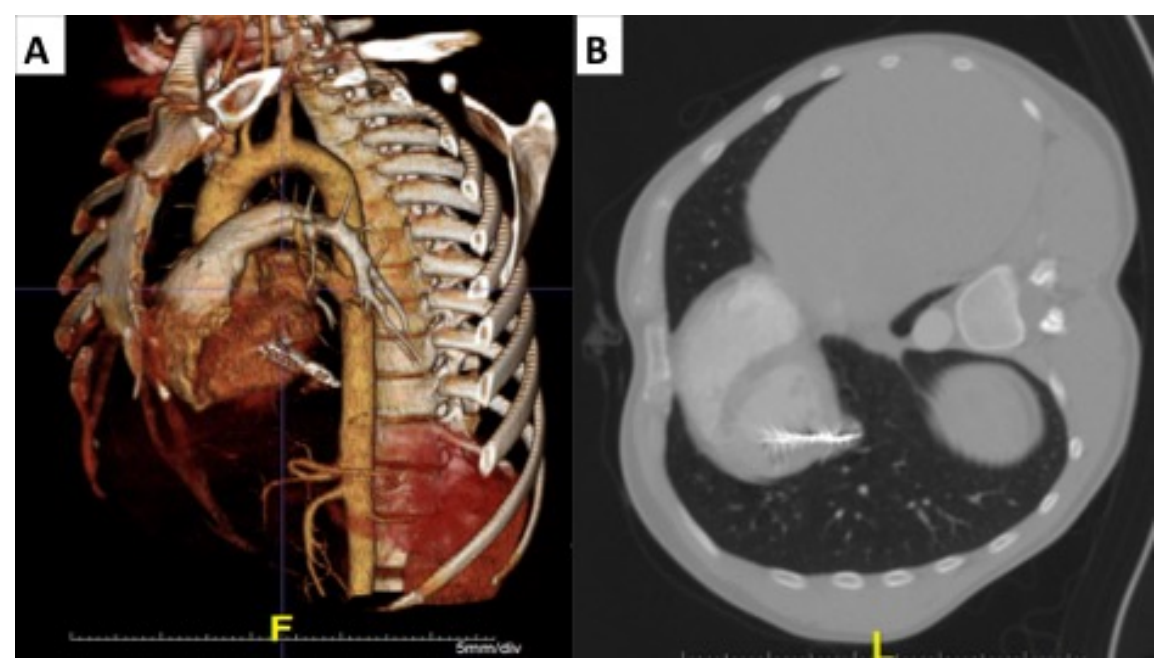

Figure 3:

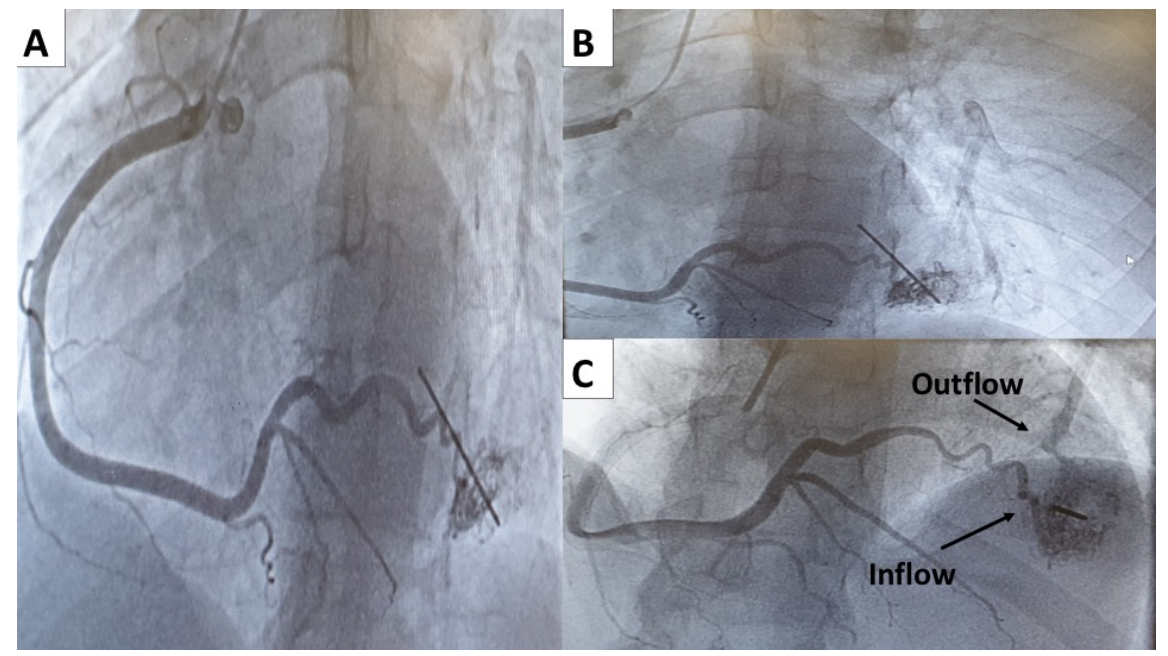

Figure 4: 


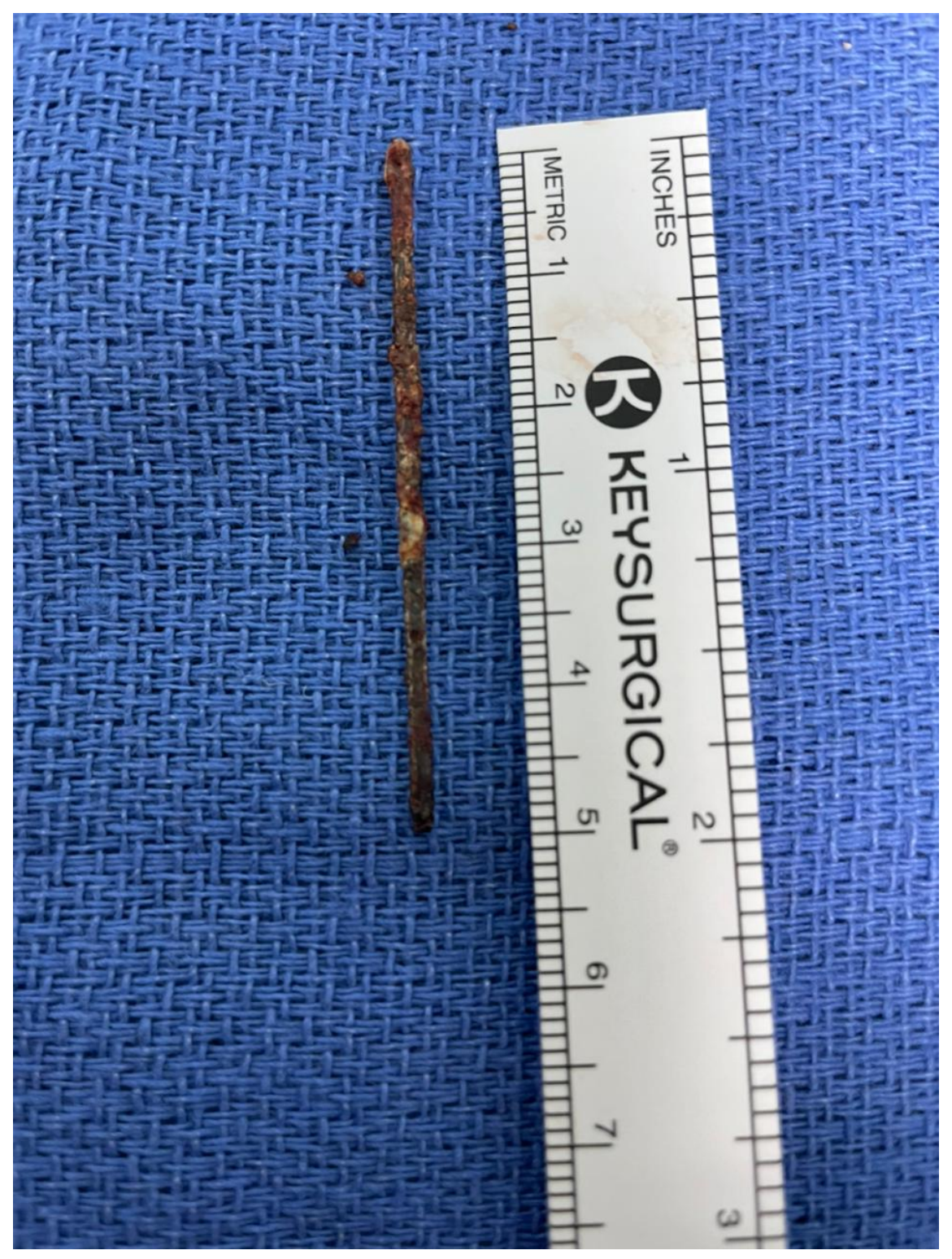

Figure 5: 

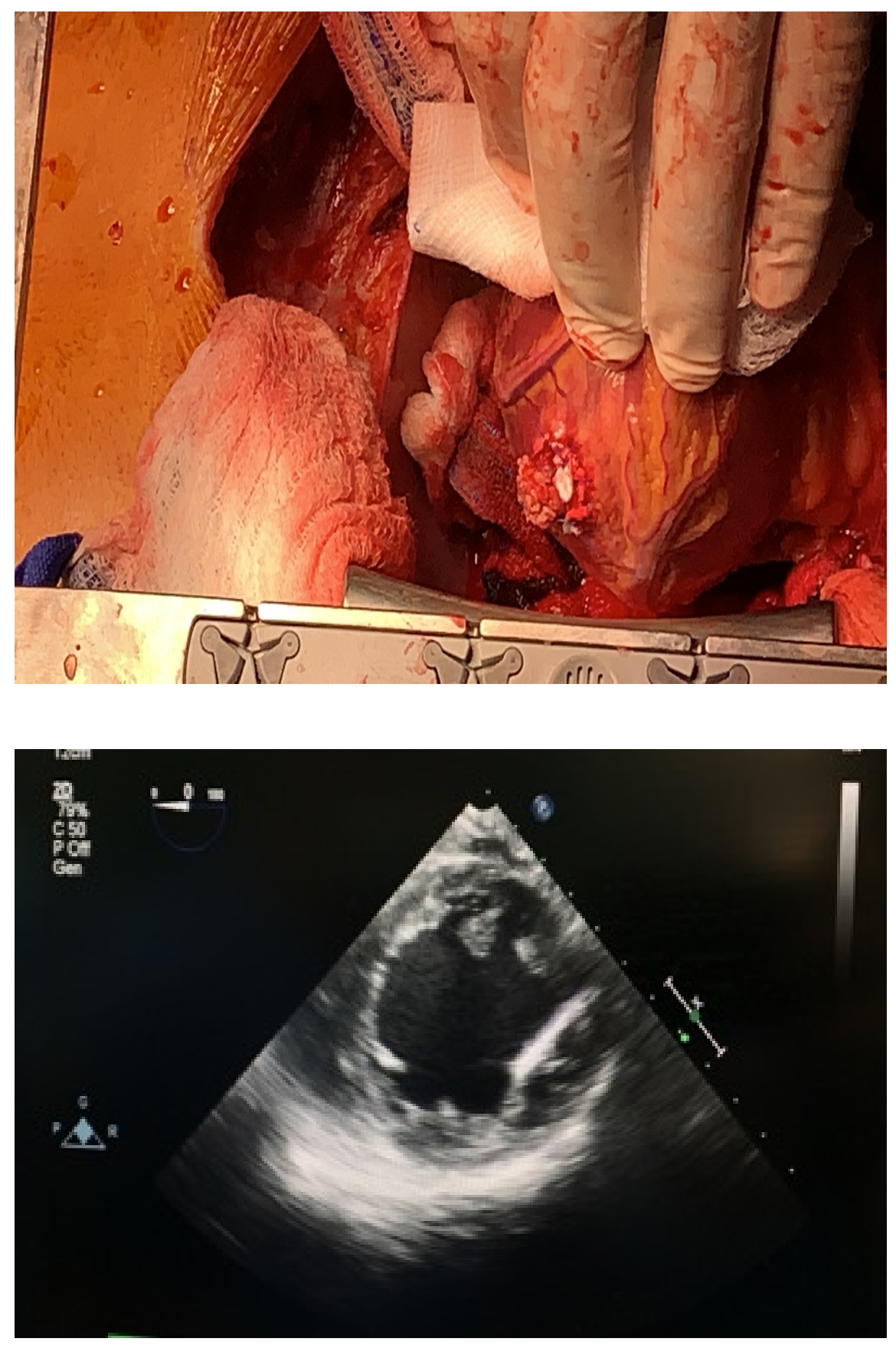


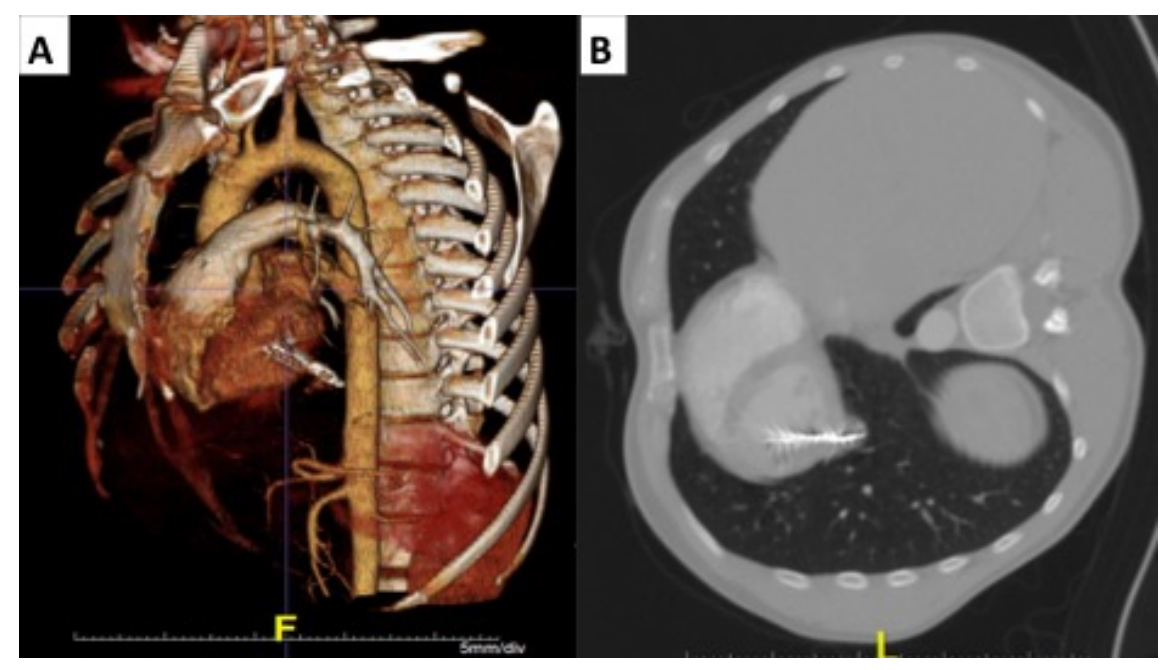

A

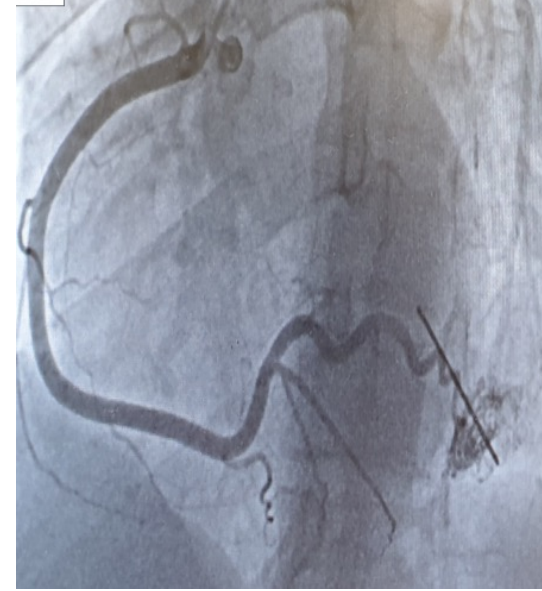

B Outflow nflow 


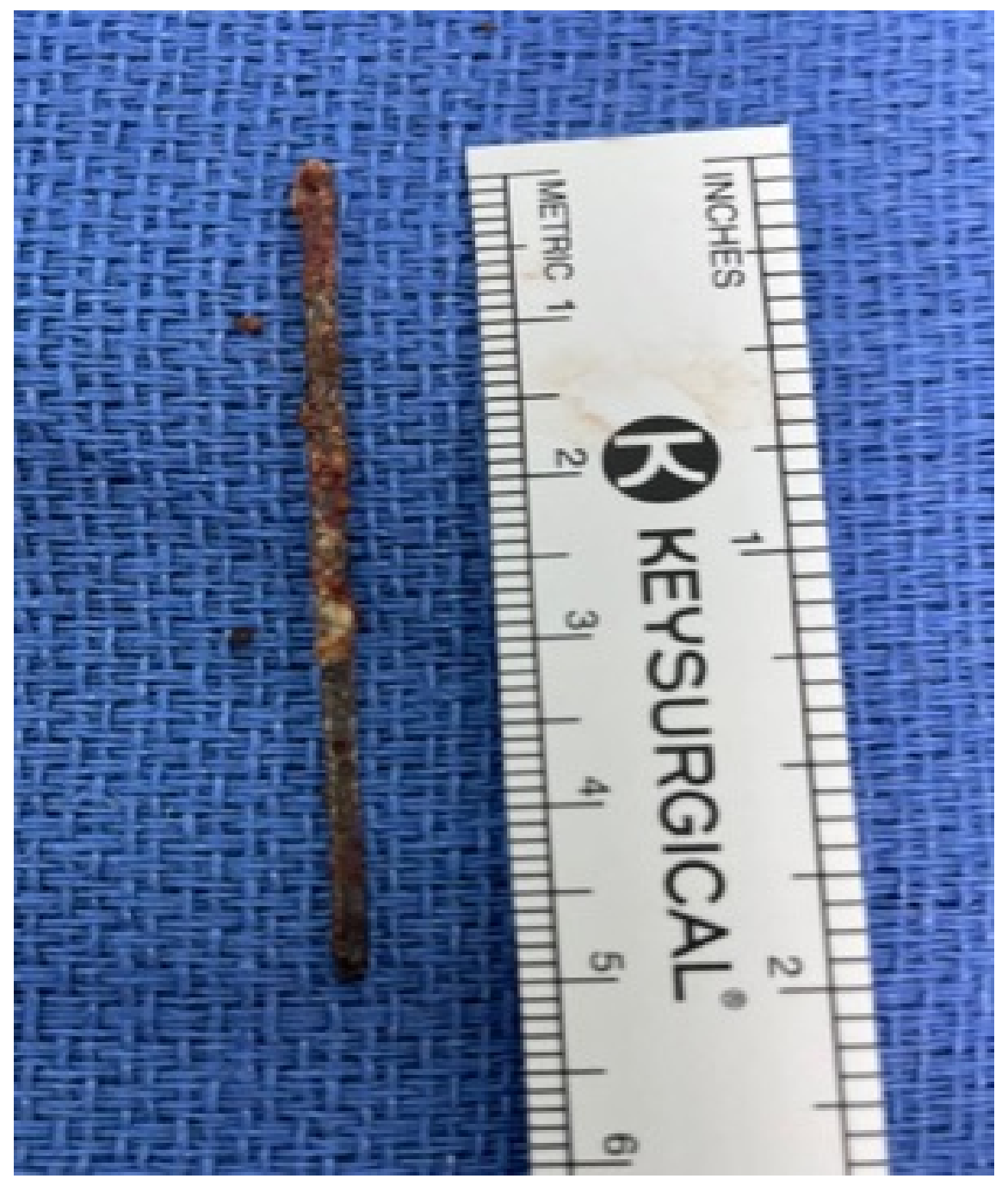




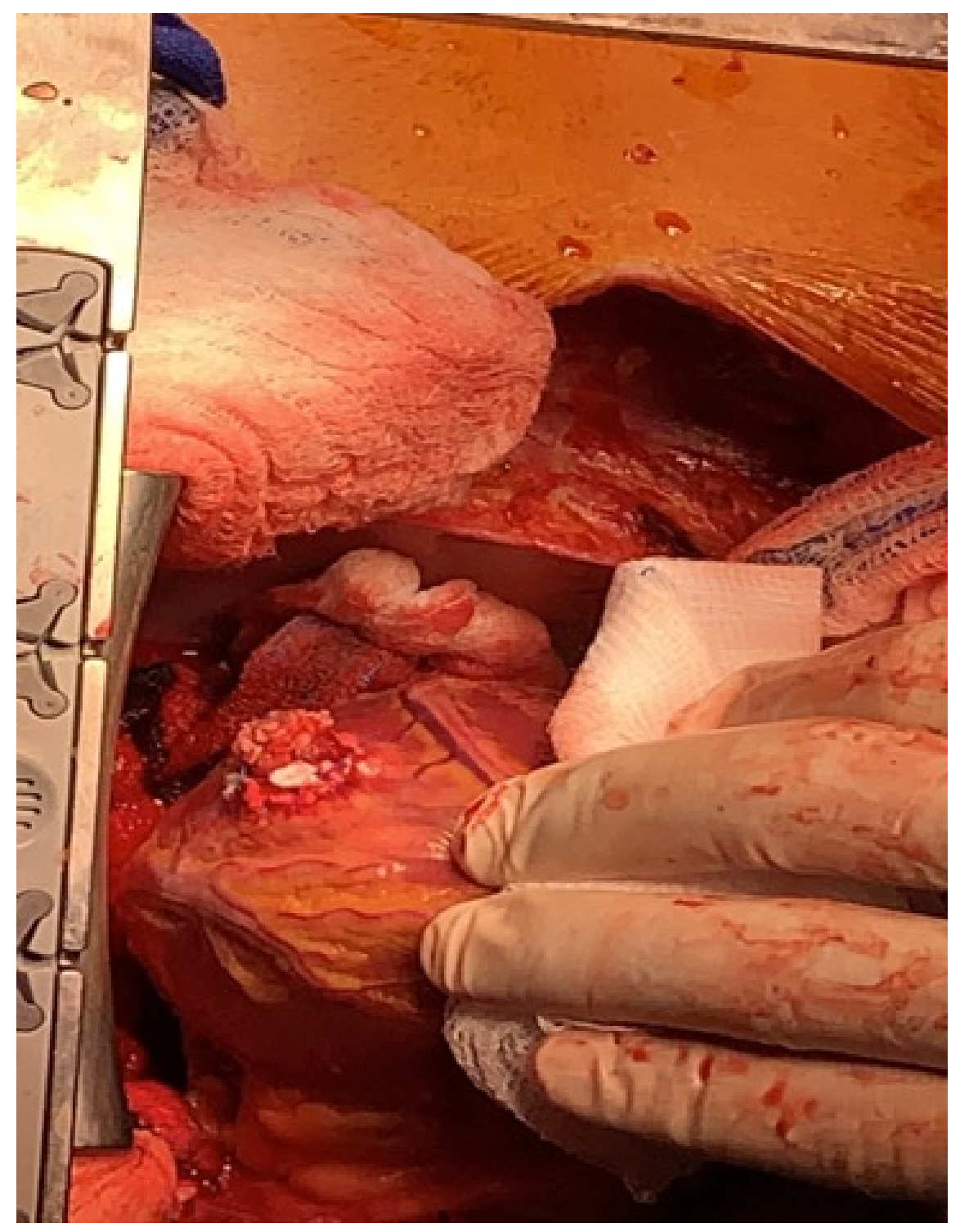

\section{Hosted file}

Table 1.pdf available at https://authorea.com/users/411026/articles/520199-nail-gun-injuryto-the-left-ventricle-causing-coronary-pulmonary-fistula-a-case-report 